\title{
Alianzas para la innovación en organizaciones intensivas en conocimiento: el caso de México*
}

\author{
Alliances for Innovation at Knowledge-Intensive Organizations: The Case of \\ Mexico
}

\author{
Juan Carlos Becerril-Elías \\ Doctorando Gestión y Políticas de Innovación, Instituto Politécnico Nacional, \\ Ciudad de México-México, jcbe05@gmail.com \\ Humberto Merritt \\ Doctor en Política Científica y Tecnológica, Instituto Politécnico Nacional, \\ Ciudad de México-México,hmerritt@ipn.mx
}

\begin{abstract}
Cómo citar / How to cite
Becerril-Elías, J. C.; Merrit, H. (2021). Alianzas para la innovación en organizaciones intensivas en conocimiento: el caso de México. Revista CEA, v. 7, n. 14, e1780. https://doi.org/10.22430/24223182.1780
\end{abstract}

Recibido: 17 de enero de 2021

Aceptado: 13 de abril de 2021

\begin{abstract}
Resumen
Las alianzas tecnológicas entre organizaciones intensivas en conocimiento enfrentan desafíos relativos a la eficiencia de la vinculación, su efectividad, su duración e incluso al impacto esperado en la innovación; además, algunas de ellas son desconocidas de forma anticipada por las organizaciones. Este artículo analizó los factores que influyen en la formación de este tipo de alianzas en el sector industrial mexicano. La hipótesis plantea que las alianzas entre organizaciones intensivas en conocimiento se ven afectadas por distorsiones, discrepancias y empirismos aplicativos debido a visiones incompletas por parte de los líderes. Para el desarrollo de este trabajo, se emplearon cuestionarios y entrevistas a actores relevantes de dos organizaciones mexicanas intensivas en conocimiento, una del sector académico y otra del sector productivo, esto con la finalidad de identificar los recursos que poseían previamente a la formalización de la alianza tecnológica y cómo sirven a la toma de decisiones. Los resultados revelaron que el rol que desempeñen los líderes tiende a robustecer la posición estratégica de las organizaciones involucradas, siempre que las actividades de investigación complementen los recursos y capacidades disponibles, sirviendo éstas para afianzar
\end{abstract}

\footnotetext{
* Este artículo se deriva del proyecto titulado «Alianzas para la innovación en organizaciones intensivas en conocimiento: el caso de México» SIP-20200079 y ha sido financiado con recursos de la Secretaría de Investigación y Posgrado del Instituto Politécnico Nacional de México.
} 
la colaboración. Se concluye que las alianzas interorganizacionales son fundamentales para lograr el éxito en el campo empresarial y en distintos sectores, sin embargo, debe tomarse en cuenta que no siempre estas asociaciones satisfacen las expectativas de los participantes.

Palabras clave: innovación tecnológica, alianzas colaborativas, conocimiento organizacional, investigación aplicada.

Clasificación JEL: L14, L24, L25, O14, O32.

\title{
Highlights
}

- Las alianzas organizacionales y tecnológicas presentan distintas dimensiones de análisis al intentar innovar.

- El modelo de análisis contiene causas y efectos mayoritarios y minoritarios del problema, es decir, distorsiones, discrepancias y empirismos aplicativos.

\begin{abstract}
Technological alliances between knowledge-intensive organizations face challenges related to the efficiency of the linkage, its effectiveness, duration, and even its expected impact on the innovation. However, organizations do not know about some of these challenges in advance. This paper analyzes the factors that influence the formation of this kind of alliances in the Mexican industrial sector. According to the hypothesis proposed here, alliances between knowledge-intensive organizations are affected by distortions, discrepancies, and application empiricisms due to the incomplete vision of their leaders. This study used questionnaires and interviews with relevant actors at two Mexican knowledge-intensive organizations (one in the academic sector and the other in the productive sector) in order to identify the resources they already had before the formalization of technological alliances and how they can support decision-making. The results revealed that the role leaders play tends to make the strategic position of their organizations more robust, as long as research activities complement the available resources and capabilities, which can consolidate the collaboration. In conclusion, inter-organizational alliances are essential for success in the business world and different sectors; however, they do not always meet the expectations of their participants.
\end{abstract}

Keywords: Technological alliance, collaborative alliance, organizational knowledge, applied research.

JEL classification: L14, L24, L25, O14, O32.

\section{Highlights}

- Organizational and technological alliances present different dimensions that organizations should analyze when they try to innovate.

- The model under analysis contains major and minor causes and effects of the problem, that is, distortions, discrepancies, and application empiricisms. 


\section{INTRODUCCIÓN}

Las alianzas interorganizacionales son un componente estratégico crítico en muchas industrias (Zhao et al., 2018; Marra et al., 2020); sin embargo, un número significativo de ellas no logra alcanzar los objetivos inicialmente propuestos (Holmberg \& Cummings, 2009). Si bien las razones detrás de las fallas son complejas y varían según el tipo de alianza e industria, muchas son resultado de estrategias deficientes surgidas de modelos teóricos inviables (Albers et al., 2013). El fracaso ocasional de alianzas interorganizacionales también obedece a una selección inapropiada de los socios debido a la carencia de mecanismos adecuados para la identificación de metas comunes. No obstante, estas fallas se podrían resolver a través de la participación informada de los líderes organizacionales en anticipación a la formalización de las posibles vinculaciones (DePamphilis, 2018; Contractor \& Reuer, 2019).

Aunque la literatura ha analizado los determinantes de la vinculación, raramente ha estudiado los elementos que definen la operatividad de las alianzas (Contractor \& Reuer, 2019), por lo que resulta pertinente preguntar: ¿qué procedimientos siguen los líderes de las organizaciones para formalizar una alianza? A este respecto, varios estudios han encontrado que el poder de negociación de los socios potenciales, las condiciones del entorno, el grado de configuración y calidad de los activos complementarios, y el grado de diversificación de los productos y servicios llevan a las organizaciones a formar alianzas (Gulati, 2007; De Man, 2014; Albers et al., 2013; DePamphilis, 2018). El impacto de estas condiciones resulta fundamental para entender la operatividad de las alianzas, pues como lo sugieren Gillespie y Gulati (2001) y Kehrel et al., (2016), una alianza involucra la intercomunicación, el intercambio o el uso común de recursos como el capital, la tecnología o las rutinas organizacionales, para lo que el mantenimiento de su estabilidad es crucial.

En este trabajo se plantea la hipótesis de que las alianzas entre organizaciones intensivas en conocimiento se ven afectadas por distorsiones, discrepancias y empirismos aplicativos debido a visiones incompletas por parte de los directivos de las organizaciones involucradas. Se argumenta que las organizaciones pocas veces discuten las precondiciones que conducen a una vinculación efectiva. Generalmente estas deficiencias surgen de una falta de visión por parte de los liderazgos, volviéndose la principal fuente de distorsiones para los objetivos de la alianza, y al haber una deficiente percepción del entorno se generan discrepancias en la forma de atender las obligaciones contraídas entre los participantes. Es decir, surgen divergencias en la interpretación de las bases teóricas que fundamentan la alianza. Finalmente, tanto las distorsiones como las discrepancias se resuelven generalmente de manera contingente, pero sin que se apliquen reglas teóricas precisas sino mediante soluciones ad hoc, condición que se denomina aquí como empirismos aplicativos, siguiendo el criterio de clasificación que sugiere Caballero Romero (2014).

El trabajo analiza dos organizaciones intensivas en conocimiento, cuyos líderes fueron los responsables de fomentar alianzas para innovar. Estas organizaciones operan en el ramo de la electrónica automotriz. La primera es un centro público de investigación y la segunda es una empresa transnacional con operaciones en México. 


\section{MARCO TEÓRICO}

\section{Las alianzas estratégicas interorganizacionales}

El estudio de las alianzas estratégicas interorganizacionales creció significativamente en los últimos veinte años a partir de investigaciones centradas en la operación de redes interpersonales (Hagedoorn, 1993; Yoshino \& Rangan, 1995; Gulati, 2007). Estos trabajos ayudaron a comprender el papel que desempeñan las redes en la configuración del comportamiento interorganizacional y de sus resultados (Gulati et al., 2011), mientras que investigaciones recientes han explorado los matices de los mecanismos subyacentes a esta interacción, profundizando en las contingencias en torno a los efectos de la vinculación (Coletti \& Landoni, 2018; Contractor \& Reuer, 2019). En el área tecnológica, el estudio de las alianzas estratégicas también ha sido ampliamente analizado. Varios trabajos coinciden en señalar que la colaboración tecnológica requiere que los participantes posean un acervo apropiado (y relativamente equivalente) de recursos y capacidades tecnocientíficas para consolidar las ventajas competitivas mutuas (García-Canal et al., 2013; Haeussler et al., 2012; Weng, 2017; Yang \& Meyer, 2019).

Por otra parte, la literatura especializada en aprendizaje y cambio organizacional ha enfatizado el papel de la empresa como repositorio de recursos (Resource-based view of the firm, en inglés), sugiriendo que los activos intangibles son la base de la ventaja competitiva de largo plazo (Muwardi et al., 2020), siendo el conocimiento organizacional el principal (Tjemkes et al., 2017; Coletti \& Landoni, 2018; Zhao et al., 2018). Así, el conocimiento organizacional, y especialmente el tecnológico, crea una ventaja sostenida, la cual es especialmente valiosa en un entorno dinámico (Belso \& Díez, 2018). Más aún, el valor añadido del conocimiento organizacional surge de factores como la singularidad del mismo (uniquess, en inglés), la especificidad de la trayectoria organizacional (pathdependency) y las características competitivas inherentes a la idiosincrasia institucional (identity) (Nooteboom, 2010).

Una característica importante de las alianzas interorganizacionales es que pueden ser horizontales o verticales (Bouncken et al., 2016), dependiendo de la relación de los participantes a lo largo de la cadena de valor, pudiéndose también clasificar de acuerdo con las motivaciones, así como por la estructura de gobernanza utilizada (Kogut, 2008). En relación con la gobernanza, las distintas opciones de operatividad crean la necesidad de evaluar la gama de acuerdos de cooperación posibles (Haeussler et al., 2012; Coletti \& Landoni, 2018). Así, para Yoshino y Rangan (1995), la gobernanza debe responder a las siguientes condiciones: 1) las organizaciones deben permanecer legalmente independientes después de que se forme la alianza; 2) deben compartir los beneficios y el control administrativo sobre el desempeño de la alianza; y 3) deben hacer contribuciones continuas. Es de esperar, entonces, que las alianzas abarquen tanto la cooperación basada en proyectos a corto plazo como la búsqueda de diversos grados de integración vertical e interdependencia y/o de sinergias horizontales.

Al responder la operatividad de las alianzas a elementos de tipo idiosincrático, su análisis se mueve hacia la dimensión intermedia de la organización. En este punto, Yasuda e lijima (2005) proponen seis temas para entender la formalización de alianzas, a saber: 1) Su formación depende de factores inductores que sirven de moduladores del proceso, 2) su estructura incluye formas de gobierno basadas en acciones y contratos, donde los efectos ambientales y tecnológicos inciden en los ciclos 
de vida de la alianza, 3) su gestión incluye el efecto del aprendizaje, siendo crucial el papel de los líderes, 4) la viabilidad de la alianza depende del desarrollo de pautas para medir el éxito y de mecanismos de identificación de las causas del fracaso, 5) el desempeño se evalúa mediante métricas específicas como los indicadores de rentabilidad vía precio de las acciones, y 6) el dinamismo de la vinculación se refleja en el nivel de transferencia de conocimiento entre los participantes, los procedimientos de aprendizaje y los mecanismos para la protección del conocimiento emanado de la alianza.

Si bien estos elementos ayudan a discernir los procesos administrativos y organizacionales que conducen la creación de alianzas, la vinculación también conlleva riesgos de conflictos e incluso pérdidas, tal y como lo apuntan Tjemkes et al. (2017), y se resume en la Tabla 1.

Tabla 1. Ventajas y desventajas de las alianzas estratégicas interorganizacionales

Table 1. Advantages and disadvantages of inter-organizational strategic alliances

Ventajas

Desventajas

\begin{abstract}
Acceso a recursos: las alianzas conllevan el acceso a Pérdida de información propietaria: información crítica capital, habilidades especializadas, entrada al mercado y puede fugarse hacia un socio que es competidor o que la tecnología e instalaciones de producción, reforzando eventualmente se convertirá en uno.

las competencias básicas.

Complejidad de gestión: las alianzas requieren del Economías de escala: los altos costos fijos requieren que las organizaciones colaboren para expandir el volumen de producción. esfuerzo combinado de los participantes, lo que complica la coordinación, pudiendo producir conflictos internos y demoras costosas.
\end{abstract}

Riesgo y costo compartido: las alianzas permiten a los Riesgos financieros y organizativos: el potencial socios compartir riesgos y costos de inversiones.

comportamiento oportunista de los socios puede

Acceso a mercados: asociarse con otra empresa es a menudo la única forma de obtener acceso a un mercado de difícil acceso, incluso en el extranjero.

socavar la lógica de creación de valor de una alianza. Las rutinas interorganizacionales también pueden dificultar que una empresa actúe de manera independiente.

Aprendizaje: las alianzas ofrecen la oportunidad de aprender prácticas de los socios, como la manufactura esbelta, el desarrollo de innovaciones y el conocimiento de la gestión de capacidades tecnológicas.

Velocidad de comercialización: los socios con habilidades complementarias agilizan la velocidad de comercialización vía ventajas de primer entrante.

Riesgo de dependencia: puede surgir un desequilibrio de poder si un socio se vuelve demasiado dependiente del otro. Esta situación aumenta el riesgo del oportunismo para realizar una adquisición hostil, por ejemplo.

Pérdida de autonomía de decisión: la planificación Reputación: las alianzas aumentan la reputación y legitimación de los socios. El cabildeo y la presión conjunta incitan al gobierno a adoptar políticas que favorezcan alianzas específicas.

conjunta y la toma de decisiones pueden dar lugar a una pérdida de autonomía y control en la toma de decisiones.

Pérdida de flexibilidad: el establecimiento de una alianza con un socio específico puede impedir la asociación con otras organizaciones.

Implicaciones antimonopolio: los beneficios de las Neutralizar o bloquear a los competidores: los socios pueden ganar competencias y poder de mercado para neutralizar o bloquear los movimientos de un competidor mediante barreras de entrada.

alianzas desaparecen si se las desafía por motivos antimonopolio. Algunos países tienen regulaciones estrictas que prohíben ciertas relaciones comerciales.

Evaluación del socio para fusión: las alianzas ofrecen una manera de conocer mejor a un posible candidato de adquisición y disminuir la asimetría de la información.

Flexibilidad: las alianzas brindan más flexibilidad que las jerarquías y los mercados porque están sujetas a menos regulación que las fusiones y adquisiciones.

Barreras de aprendizaje: aunque las alianzas brindan acceso al conocimiento, las barreras de aprendizaje pueden dificultar la integración y la explotación de nuevos conocimientos.

Viabilidad a largo plazo: a pesar de la existencia de acuerdos y fechas de expiración, diversas contingencias pueden derribar de forma prematura la alianza.

Fuente: elaboración propia a partir de (Tjemkes et al., 2017). 
En este trabajo se propone un modelo que sintetiza los factores antes expuestos y que estimulan a las organizaciones intensivas en conocimiento a establecer alianzas colaborativas. Su objetivo es proporcionar elementos de análisis para entender la colaboración tecnológica a partir de la convergencia de elementos organizacionales. El modelo se apoya en tres componentes. El primero surge de la percepción propia de la organización, donde las racionalidades que aplican los líderes sirven de base para identificar la viabilidad de los procesos interorganizacionales destinados a fomentar la innovación. La identificación de esta dimensión es crucial porque una conceptualización divergente de la realidad puede debilitar las perspectivas de la alianza. Al respecto, Álvarez (2015) plantea la existencia de tres tipos de racionalidades imperantes entre los directivos: 1) racionalidad técnica, 2) racionalidad económica y 3) racionalidad social. Así, la interacción efectiva de estos tres componentes les facilita a los líderes la toma de decisiones, al tiempo que les genera menos incertidumbre.

El segundo componente del modelo se relaciona con los recursos internos existentes. En este punto, el estudio del conocimiento organizacional como activo intangible ha sido abordado por Prahalad y Hammel (1990), Levinthal y Myatt (1994) y Teece et al. (1997). Sus análisis se apoyan en criterios de complementariedad y sinergia, los cuales son útiles para la medición de los recursos y capacidades contenidos en cada organización. En relación con la formación de capacidades, Levinthal y Myatt (1994) sostienen que la evolución de éstas está íntimamente ligada con la evolución de los mercados en que interactúa la organización, ya que aquellas pueden crecer, refinarse, o incluso decaer dependiendo del dinamismo del mercado. Es importante resaltar que la formación de capacidades deja de ser endógena cuando el eje de referencia para la toma de decisiones no es la organización. Por su parte, Collis (1994) propone una taxonomía para medir la formación de capacidades categorizándolas en tres niveles. El primero está formado por el grupo de capacidades que reflejan la habilidad para desempeñar actividades básicas de la empresa, la segunda categoría corresponde a la mejoría de las actividades de la empresa, la cual Teece et al. (1997) describen como una cualidad de la organización para aprender, adaptarse, cambiar y renovarse en el tiempo.

El último componente descansa sobre la habilidad de la organización para concebir, cambiar e implementar estrategias. Estas características han sido descritas por Barney (1991), quien señala que las capacidades organizacionales no pueden ser transferidas de forma independiente, sino que deben ser desarrolladas y moldeadas internamente por los propios recursos humanos de la organización. Aunque esta es una característica endógena a la organización, paradójicamente las capacidades organizacionales están íntimamente ligadas al mercado, siendo condicionadas por dinámicas endógenas y exógenas en los ecosistemas de innovación donde se desenvuelven las organizaciones.

Los constructos que sirven para caracterizar las organizaciones susceptibles de formar alianzas con las miras de innovar se muestran en la siguiente Tabla donde las dimensiones internas e intermedias son las variables explicativas de la investigación, que son a su vez, parte del modelo teórico de referencia y catalogadas como causas principales del problema

\section{METODOLOGÍA}

La metodología del trabajo se basó en un enfoque de tipo cualitativo para facilitar la recopilación de la información empírica. La ventaja de la metodología cualitativa es que permite uniformar las percepciones de los agentes bajo investigación, armonizando y sistematizando las diversas 
interpretaciones, lo que permite analizar las relaciones de significado que se producen en un contexto determinado (Creswell \& Plano Clark, 2017). Es importante precisar, como lo apuntan Bund et al. (2015), que la investigación cualitativa no se esfuerza en buscar la representatividad muestral de sus resultados, ya que adquiere validez externa a través del trabajo de campo y la triangulación de resultados. Las técnicas cualitativas que se aplicaron consistieron en la realización de entrevistas, cuestionarios y observaciones directas. Las etapas metodológicas se describen a continuación.

La primera etapa consistió en el seguimiento del trabajo documental existente para conocer el estado del arte y construir el modelo que perfilaría las variables del marco de referencia teórico. A partir de esta revisión se definieron los conceptos básicos del problema de investigación: a) las distorsiones, b) las discrepancias y c) los empirismos aplicativos.

En la segunda etapa se desarrolló un trabajo exhaustivo documental para construir el marco referencial que sirvió para definir las variables del marco de referencia (causas mayoritarias del problema). Esta conceptualización permitió operativizar la hipótesis de investigación; es decir, se identificaron los liderazgos en cada organización y se contabilizaron las capacidades y los activos intangibles en las organizaciones bajo análisis con el objetivo de priorizar los criterios de validación.

En la tercera etapa se identificaron dos organizaciones intensivas en conocimiento con operaciones en México a través de la técnica de muestreo por caso. Estas instituciones se seleccionaron debido a que contaban con capacidades tecnológicas significativas en el área de la electrónica, específicamente en hardware y software, con énfasis hacia el sector automotriz. Las organizaciones son Cidetec (Centro de Investigación y Desarrollo Tecnológico en Cómputo) del Instituto Politécnico Nacional de México y Honeywell México (empresa multinacional con operaciones en la República Mexicana).

En la cuarta etapa se documentaron los antecedentes administrativos y organizacionales de ambas instituciones, enfocando la investigación hacia apreciaciones descriptivas de los datos recolectados para mejorar la percepción de las variables de realidad (liderazgos y organizaciones), dadas las limitaciones de la investigación en tiempo y recursos financieros.

En la quinta etapa se elaboraron los instrumentos de validación que fueron aplicados durante la investigación de campo. El primero fue el cuestionario, que además tuvo el mayor peso para este trabajo al utilizarse para la recolección extensa de información en ambas organizaciones, con la intención de obtener evidencia escrita de las dimensiones de interés (véase Tabla 2). El segundo instrumento, que además complementó al cuestionario, fueron las entrevistas semi estructuradas, usadas también para recopilar la información susceptible de escapar al primer instrumento.

Tabla 2. Dimensiones de validación para las organizaciones bajo análisis

Table 2. Validation dimensions for the organizations under analysis

\begin{tabular}{|c|c|c|c|c|}
\hline Actor & $\begin{array}{c}\text { Tópicos para los } \\
\text { líderes }\end{array}$ & $\begin{array}{l}\text { Tópicos para la } \\
\text { organización }\end{array}$ & $\begin{array}{c}\text { Tópicos para las } \\
\text { capacidades }\end{array}$ & $\begin{array}{c}\text { Tópicos hacia las } \\
\text { alianzas }\end{array}$ \\
\hline $\begin{array}{c}\text { Centro de } \\
\text { Investigación } \\
\text { (Cidetec) } \\
\text { Empresa } \\
\text { (HONEYWELL) }\end{array}$ & $\begin{array}{c}\text { Visión } \\
\text { Perspectiva } \\
\text { Enfoque } \\
\text { Características de } \\
\text { liderazgo }\end{array}$ & $\begin{array}{l}\text { Estructura } \\
\text { Sector } \\
\text { Herramientas } \\
\text { Estrategias }\end{array}$ & $\begin{array}{c}\text { Recursos } \\
\text { Capacidades } \\
\text { Competencias }\end{array}$ & $\begin{array}{l}\text { Disposición } \\
\text { Enfoque } \\
\text { Prioridades } \\
\text { Efectividad }\end{array}$ \\
\hline
\end{tabular}

Fuente: elaboración propia. 
Los instrumentos referidos fueron aplicados al director de proyecto y al subdirector en cada organización. Cabe señalar que la información recolectada puede ser solicitada directamente a los autores para consulta académica, pero se debe obtener la autorización previa de los interesados para garantizar los acuerdos de confidencialidad establecidos con ellos durante la investigación.

La última etapa consistió en el trabajo de escritorio donde se concentró, organizó y revisó la información recolectada en el trabajo de campo. En esta etapa se revisaron los hallazgos para validar la consistencia de los datos, buscando alcanzar la mejor descripción de las variables del modelo considerando las limitaciones de la investigación. En la siguiente sección se presentan los resultados obtenidos.

\section{RESULTADOS}

En este apartado se reportan los resultados recopilados en el trabajo documental y de campo. La selección de las unidades de análisis se basa en que ambas organizaciones son intensivas en conocimiento, distinguiéndose por estar en sectores distintos: el académico y el empresarial. Aunque las dos organizaciones poseían capacidades y vocaciones diferentes para el desarrollo de tecnologías, ambas presentaron convergencias en tecnologías de hardware y software, lo que presupone la existencia de condiciones iniciales adecuadas para el establecimiento de una alianza con miras a promover la innovación tecnológica. El análisis se documentó a partir del origen, estructura, línea de productos y servicios de la empresa Honeywell, mientras que para el Cidetec se documentó su origen, estructura y líneas de investigación científica y tecnológica.

\section{Caracterización documental de Honeywell}

De acuerdo con la información obtenida de Honeywell (2021), la empresa comenzó operaciones en México en el año 1936, contando con más de 12000 empleados al momento de la investigación. Honeywell México poseía 15 diferentes plantas de fabricación, enfocándose en actividades para la solución de seguridad y productividad. La evaluación de su estructura organizacional, a partir del diagnóstico de campo, identificó capacidades notables para los procesos logísticos, los desarrollos tecnológicos y el desarrollo de soluciones a la medida del cliente, apoyándose en el manejo de capital humano especializado. En términos de sustentar ventajas competitivas, se observa la utilización de estrategias de enfoque y de costos, está ultima para aprovechar economías de escala a la Porter (1990), lo que refleja la orientación internacional de la compañía. Además, el uso simultáneo de estas estrategias demuestra la complejidad de los procesos de la organización, como lo evidencia la gama de productos y servicios que ofrece esta firma. De acuerdo con información de su página web (https://www.honeywell.com/), la empresa multinacional tiene una línea de productos en el sector electrónico de actuadores, interruptores y sensores que comprende más de 50000 diseños distintos desarrollados a través de las capacidades de ingeniería que tiene la firma en el ámbito global. A partir de esta información se pudo comprobar que sus soluciones de interruptores y sensores cubren una gama de aplicaciones básicas y complejas, su gama de productos incluye interruptores de acción rápida, manuales y de presión, y sensores de posición, de velocidad y de flujo de aire, entre otros.

El análisis indicó que los sectores en que se ha especializado esta empresa comprenden actividades de alta tecnología, como productos aeroespaciales y de defensa, aparatos médicos, para la industria 
del petróleo, del gas y del transporte. De la revisión se detectó la presencia de una fuerte transversalidad de mercados, lo que reforzó varios procesos especializados que se derivaron en notables capacidades tecnológicas extensibles para la compañía en varios mercados.

\section{Caracterización documental de Cidetec -IPN}

El Centro de Innovación y Desarrollo Tecnológico en Cómputo del IPN ([Cidetec], 2021) nace en 1987 cuando se creó el Centro de Investigación Tecnológica en Computación (Cintec), y al año se estableció el programa de Maestría en Ingeniería de Cómputo, el cual fue aprobado por el Consejo General Consultivo del IPN, permaneciendo adscrito al Cintec hasta 1996. En 1997, el Cintec se transforma en el Cidetec, teniendo como objetivos consolidar la infraestructura de cómputo del IPN y el aprovechamiento de las ventajas tecnológicas adyacentes. Una de sus ventajas más sólidas radica en la educación profesionalizante en informática y computación. Al momento de la investigación, la estructura organizacional constaba de la dirección general, el colegio de profesores, el decanato, el comité interno de proyectos, la coordinación de enlace y gestión técnica, la unidad de informática, dos subdirecciones, cuatro departamentos distribuidos en las dos subdirecciones y una unidad de integración social y otra de tecnología educativa y campus virtual.

Las líneas de estudio, basadas en sus capacidades de investigación y recursos intangibles son: computación inteligente, mecatrónica, procesamiento paralelo, realidad virtual, redes y seguridad informática e información cuántica. Estas líneas refuerzan a los sectores automotriz y de tecnologías de la información y las comunicaciones. Actualmente, el capital humano del centro es de 25 docentes de tiempo completo, con solo una clasificación rankeado en el Sistema Nacional de Investigadores (SNI) nivel III, uno más SNI II, once elementos pertenecientes al SNI I y cuatro más dentro del SNI C, de acuerdo con su sitio web. De su oferta educativa destacan el doctorado en ingeniería de sistemas robóticos y mecatrónicos y la maestría en tecnología de cómputo.

\section{Aplicación del modelo teórico en campo}

Los resultados del trabajo de campo se obtuvieron de fuentes primarias, cuestionarios y entrevistas semiestructuradas, las cuales sirvieron para determinar las condiciones sobre las que operan los directivos en la cotidianidad, lo que significa que sirvieron para tener una observación directa sobre las visiones de los líderes de las organizaciones intensivas en conocimiento analizadas, que fueron establecidas en el modelo de análisis como las variables de la realidad. Asimismo, y siguiendo el modelo propuesto, los cuestionarios y entrevistas proporcionaron las ponderaciones internas e intermedias sobre las variables de la realidad (líderes y organizaciones) que también sirvieron para determinar las condiciones de las variables identificadas como los efectos del problema (y eje del análisis): distorsiones, discrepancias y empirismos aplicativos (Ver Figura 1). 


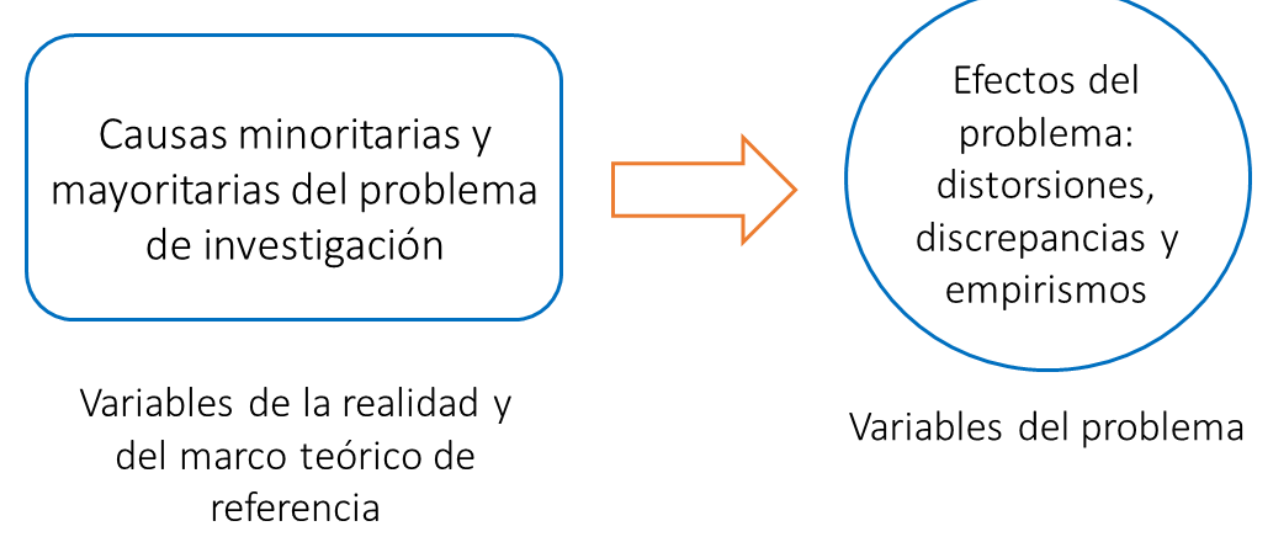

Figura 1. Definición de variables del problema (efectos)

Figure 1. Definition of the variables in the problem (effects) Fuente: elaboración propia.

\section{Evaluación de las racionalidades (causas) y distorsiones (efectos)}

En las organizaciones estudiadas, los perfiles de director y subdirector corresponden al área tecnológica que, según Álvarez (2015), encajan en el concepto de racionalidades basado en líderes con formación técnica. Las entrevistas muestran que ambos líderes declararon haber cumplido con los objetivos del puesto al alcanzar las metas de eficiencia y eficacia en el ámbito económico de sus organizaciones. Sin embargo, los entrevistados demostraron carecer de fuertes habilidades notécnicas (situación referida como racionalidad humanista y económica) porque mencionaron tener anhelos de exploración en áreas no afines a su formación, como son las ciencias sociales, administrativas, financieras y de análisis estratégico. Este efecto de búsqueda de complementariedad, consciente o inconsciente, puede ser un motor de la interacción continúa con otros actores y a la postre generador de alianzas, además de denotar la posibilidad de distorsiones en la operación de la organización por parte de los líderes. Lo anterior derivado de los sesgos que las racionalidades profesionales detonan en la visión del líder y en las decisiones sobre la organización. Este fenómeno puede considerarse, en parte, como un proceso de retroalimentación positiva, donde las visiones de los lideres influyen en la adquisición de recursos estratégicos y la construcción de nuevas capacidades (coevolución) (Levinthal \& Myatt, 1994).

Los resultados reportados sobre racionalidades y objetivos cumplidos (y por alcanzar) muestran que las distintas racionalidades de los líderes favorecen acciones en una dirección de crecimiento organizacional y de formación de alianzas, mientras que inhiben otras. Las entrevistas sirvieron para validar el constructo denominado "discreción gerencial," que ha sido descrito por Levinthal y Myatt (1994). La existencia de este comportamiento tiende a causar distorsiones en la elección de formación de capacidades y en la implementación de las estrategias organizacionales.

\section{Recursos y capacidades(causas), distorsiones y discrepancias (efectos)}

Los recursos y capacidades de las organizaciones investigadas mostraron ser dispares, pues mientras la empresa Honeywell (2021) accede a una red mundial con ingresos de 25 millones de dólares anuales, Cidetec solo dispone de una bolsa variable de recursos económicos anuales de entre 1 y 10 
millones de pesos mexicanos. De acuerdo con las entrevistas, esta disponibilidad de recursos se relaciona de manera directa con los efectos creados por las distorsiones, las discrepancias y los empirismos que desarrollan los líderes de estas organizaciones, produciendo consecuencias tales como un menor dominio de herramientas administrativas y una infraestructura mucho menor, lo que genera un entorno deficiente para las alianzas e inhibe la posibilidad de iniciar proyectos innovadores.

En relación con el tema del capital humano disponible, se debe apuntar que una ventaja competitiva para los centros de investigación es poder disponer de capital humano de forma abundante, pues aprovechan la formación de investigadores. Sin embargo, los datos recolectados sugieren grandes diferencias entre ambas organizaciones en cuanto a su plantilla de expertos. Por ejemplo, Cidetec, aunque tiene menos personal, posee (en promedio) un mayor nivel académico, pues sus docentes contaban con posgrados (maestría y mayoritariamente doctorado). En contraste, los responsables del desarrollo tecnológico en Honeywell solo exhibieron tener una preparación básica en ingeniería, sin mayor calificación académica posterior.

Aunque Cidetec mostró tener el mayor nivel académico, esto no pareció potenciar la generación de productos y/o servicios innovadores después de revisar el número de patentes registradas y el número de nuevos productos reportados, pero sí resultó un factor determinante para detonar la alianza sinérgica con Honeywell, de acuerdo con los entrevistados. En todo caso, es Honeywell la que obtuvo un mayor dinamismo innovador, ya que registró 18 innovaciones de producto/proceso por trimestre (en promedio), mientras Cidetec reportaba solo 6 productos desarrollados de forma anual. De acuerdo con lo anterior se puede ver que el nivel de conocimiento académico (por sí solo) no propicia que centros de investigación como Cidetec generen innovaciones; aunque estar en contacto con organizaciones con mayores recursos y capacidades de mercado sí pueden incentivar la búsqueda de innovaciones, empero de un menor nivel académico por el perfil de los equipos de especialistas.

Adicionalmente, si se consideran las capacidades tecnológicas como la suma de los propios procesos tecnológicos, más el capital humano que los diseña, implementa y ejecuta, la evidencia recolectada sugiere que Honeywell cuenta con mayores capacidades para innovar al tener más recursos, implementar estudios y utilizar herramientas de la gestión de la tecnología para la toma de decisiones, lo que le permite mejorar sus posibilidades de innovar en productos y en procesos. Sin embargo, el desconocimiento de algunas técnicas y/o herramientas para generar análisis más diversificados pueden ser una fuente de distorsiones aún para los líderes de esta empresa transnacional.

\section{Dimensión intermedia (causas), discrepancias aplicativas y distorsiones (efectos)}

Para evaluar las capacidades de innovación y vinculación se formuló la siguiente pregunta: ¿con qué frecuencia se vincula con otras organizaciones? Las dos organizaciones reportaron una frecuencia de más de 5 colaboraciones al año, resultado condicionado, en cierta medida, por las limitaciones de las propias estructuras organizativas y del tipo de liderazgo derivado de los ciclos de gestión de directores.

Para describir la efectividad de las alianzas se les preguntó lo siguiente: 1) ¿cuántas vinculaciones de las que realiza en el año son efectivas o dan resultados tangibles? y 2) ¿̇en cuánto tiempo se obtienen 
resultados de la vinculación hecha por su organización? De acuerdo con los entrevistados, en Honeywell la tasa de impactos de vinculaciones fue del 20 por ciento al año, mientras que Cidetec declaró tener una tasa de al menos 40 por ciento. El tiempo que Honeywell empleó para obtener resultados de estas vinculaciones fue entre 12 y 18 meses debido a restricciones internas de efectividad. En plazos menores a 12 meses, el desarrollo tecnológico es simple y de poco impacto y en plazos mayores a 18 meses los trabajos en grupo se vician y no logran los objetivos planteados, según reportó el entrevistado. En este rubro el Cidetec declaró tener un tiempo promedio de 12 meses para obtener resultados efectivos de las vinculaciones. Estas respuestas denotan fenómenos relacionados con las distintas formas de aplicar procesos de innovación (discrepancias) entre ambas organizaciones.

Por otra parte, la evidencia de que existen distorsiones y discrepancias por parte de los líderes de estas organizaciones surge al indagar sobre lo que buscan obtener cada una cuando establecen alianzas. La convergencia en las respuestas de los entrevistados de Honeywell y Cidetec indica el interés sobre la obtención de nuevo conocimiento e información como principal insumo para establecer alianzas, mientras que la divergencia de intereses sobre las dimensiones de nuevos mercados, tecnología, financiamiento y capital social y relacional es también evidente. Esto resulta del efecto de las distorsiones de liderazgos y discrepancias entre las dos organizaciones, tal y como se adelantó con el modelo de análisis y las variables del problema definidas al inicio. La Figura 2 muestra las coincidencias y divergencias de los líderes en las dos organizaciones respecto a 5 temas estratégicos.

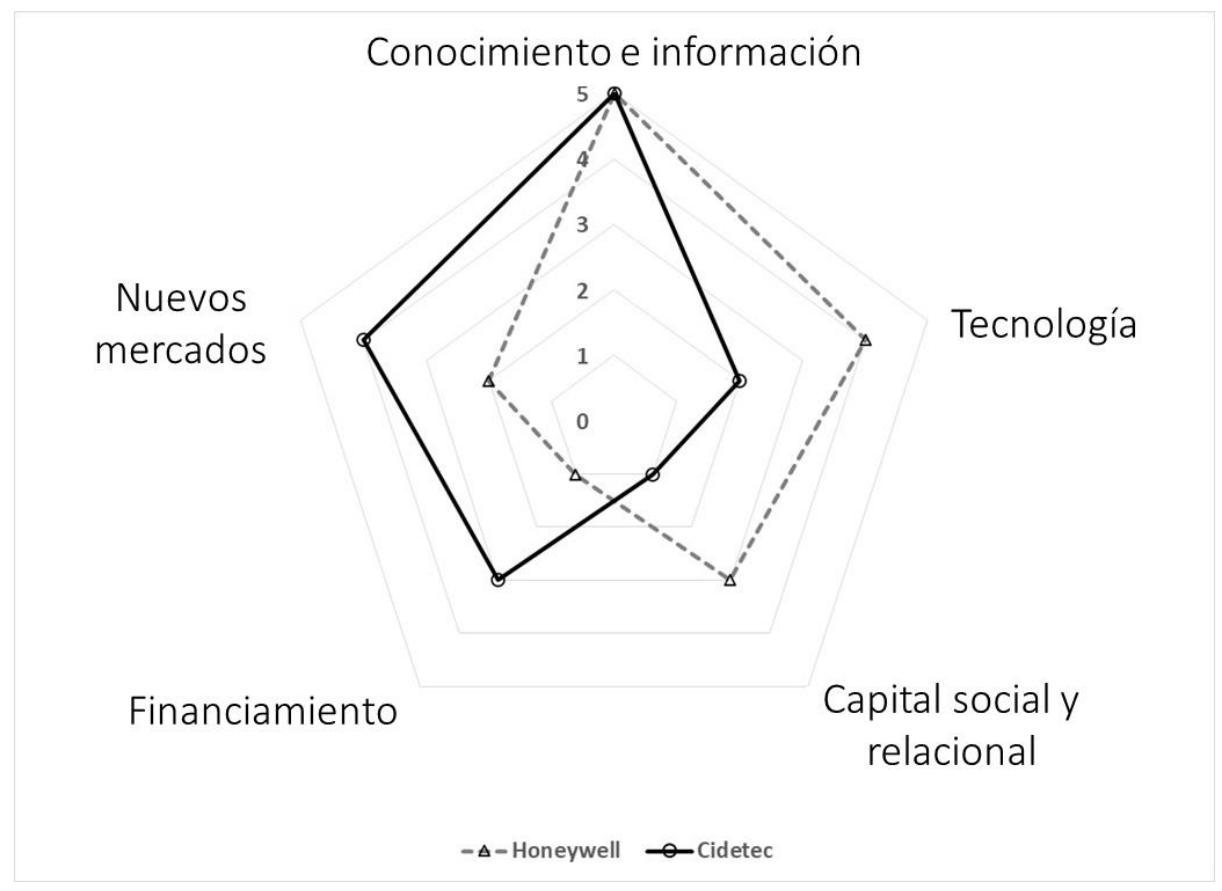

Figura 2. Valoración de los intereses declarados por las organizaciones

Figure 2. Scores of the interests expressed by the organizations

Fuente: elaboración propia.

Nota: la escala es Likert, donde 5 el más importante y 1 el menos importante. 
De acuerdo con Pavitt (2005), existen tres subprocesos en la innovación: a) producción de conocimiento, b) transformación de conocimiento en artefactos o productos, sistemas, procesos y servicios y c) cruce continuo con las necesidades y demandas del mercado. Al analizar la interpretación que de estos subprocesos hacen los líderes de las dos organizaciones, se observó que reconocieron el primer subproceso como altamente relevante para sus estructuras, pues asignan la evaluación más alta en el interés de intercambio. Para el segundo subproceso, Honeywell mostró un mayor interés en poder realizar la conversión de conocimiento en productos, servicios, procesos y sistemas, reportando 18 innovaciones en producto o en proceso por trimestre (en promedio), mientras que Cidetec reportó 6 productos desarrollados de forma anual. Mediante el trabajo de campo se identificó que el fenómeno de las discrepancias aplicativas en el proceso de innovación subsistía en ambas organizaciones. Por último, para el tercer subproceso de la innovación, dada su orientación comercial, Honeywell mostró una mayor cercanía al mercado, pero por la misma situación de ser una meta cubierta internamente, la empresa mostró poco interés en reforzar más su interacción con el mercado, sugiriendo una fuente adicional de distorsiones y discrepancias para el liderazgo de cada organización.

La importancia de la vinculación se realizó midiendo el valor concedido por los entrevistados a los actores con quienes establecieron algún tipo de colaboración. La evidencia indica que existen diferencias entre los líderes que pueden ser interpretadas, en parte, por la diferencia en el grado de adaptación de las organizaciones a entornos, tanto estables como turbulentos, tal y como lo plantean Crozier y Friedberg (1990). En el caso de Honeywell su prioridad fue vincularse con universidades y centros de investigación, mientras que Cidetec tuvo como principal socio al Gobierno. Es de resaltar que la sociedad civil no fue un colaborador relevante para ninguno de los dos, según la Figura 3.

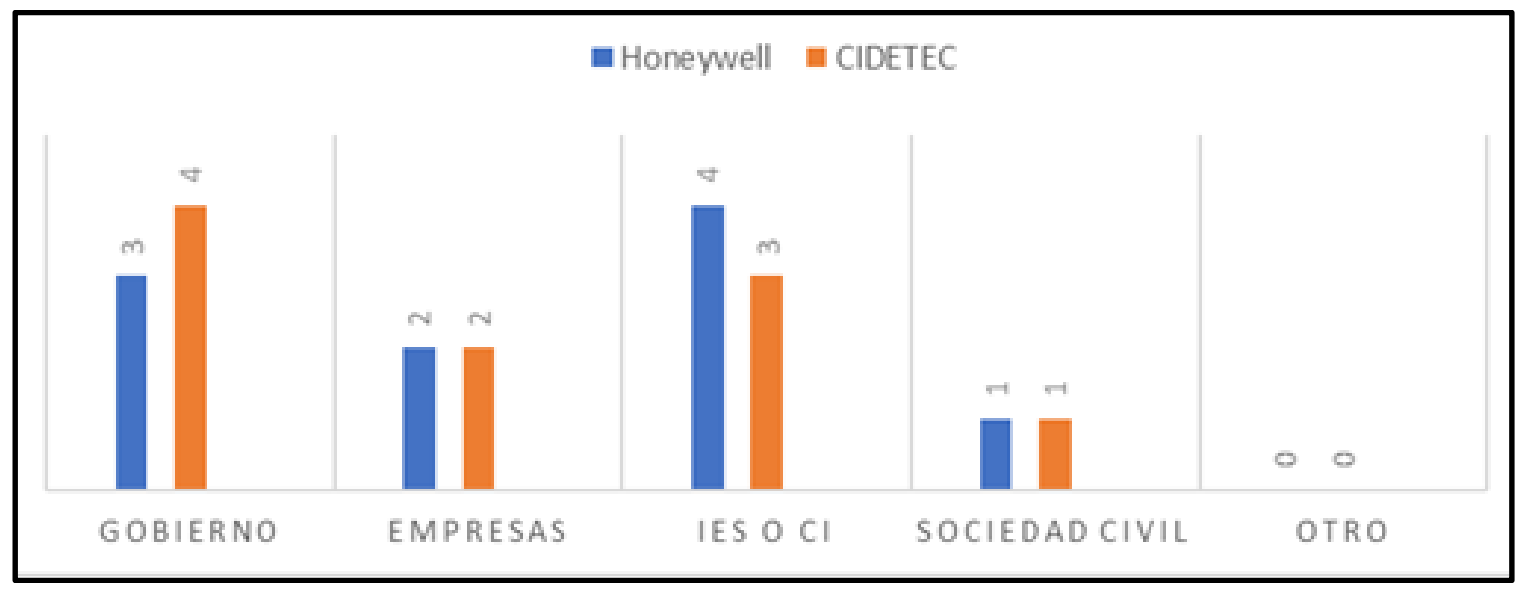

Figura 3. Principales socios para vinculación

Figure 3. Most common linkages of the organizations under analysis Fuente: elaboración propia.

Nota: la escala es Likert, donde 4 el más importante y 1 el menos importante.

El grado de eficiencia en las alianzas se abordó al formular la pregunta siguiente: ¿cuántas mejoras a servicios, productos o procesos han surgido a partir de la colaboración con otras organizaciones? Los entrevistados dijeron que Honeywell registró que 80 por ciento de las colaboraciones produjo al menos un producto o proceso innovador, mientras que Cidetec realizó 4 alianzas por año, de las 
cuales surgieron 66 por ciento de los productos generados durante el mismo periodo. La diferencia se explica, de alguna forma, por los mayores recursos y capacidades de la empresa, así como a las diferencias en el grado de discreción gerencial (i.e., toma de decisiones), que tendieron a afectar el desempeño de la dimensión intermedia de análisis, lo que demuestra la mayor relevancia de las variables de la dimensión interna que las variables de la dimensión intermedia del modelo.

Por otra parte, la percepción de los tomadores de decisiones de las organizaciones analizadas respecto a los riesgos que buscaron minimizar al establecer sus alianzas, arrojó los siguientes datos. La empresa Honeywell tiene como prioridad la disminución de tiempos de aprendizaje organizacional (variable interna del modelo), mientras que el centro de investigación del IPN tiene como prioridad la disminución de costos (elemento relacionado con los recursos financieros). En contra parte, para Honeywell no fue importante el riesgo de mercado debido a que forma parte de su entorno cotidiano. Para Cidetec la disminución de los tiempos de producción no resultó de importancia porque su condición de entidad pública la hizo inmune a las condiciones de mercado. De estos datos se puede inferir que la naturaleza económica de la empresa hace que pierda interés en factores en los que (aparentemente) ya tenía dominio, al igual que sucedió con la naturaleza institucional pública del Cidetec sobre los tiempos de producción que se le imponían. La Figura 4 resume estos hallazgos.

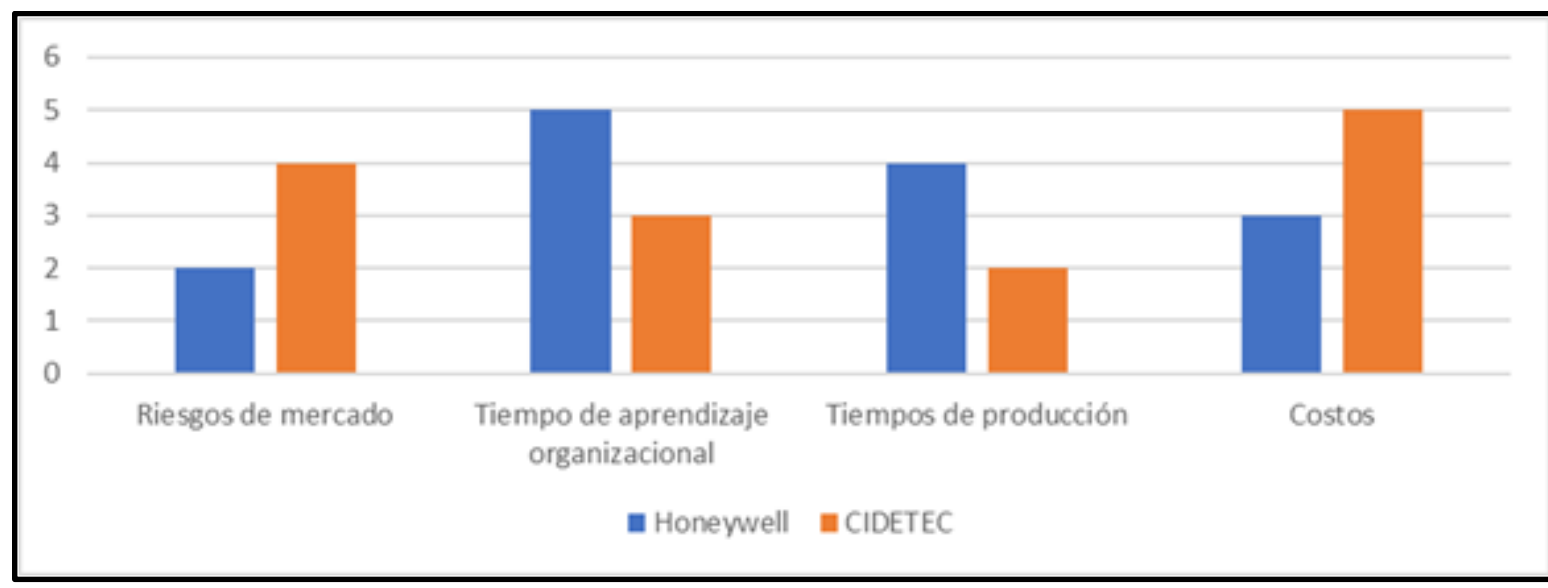

Figura 4. Grado de importancia en los factores que determinan la vinculación

Figure 4. Importance of the factors that determine the linkages Fuente: elaboración propia.

N Nota: la escala es Likert, donde 5 el más importante y 1 el menos importante.

Otro punto de interés fue indagar sobre los factores que pueden maximizar la probabilidad de establecer alianzas. Del trabajo de campo se desprende que Cidetec manifestó mayor interés en explotar su conocimiento acumulado. Por su parte, Honeywell (desde la perspectiva de los entrevistados) buscó maximizar la rentabilidad de su propiedad intelectual. Con base en esto se puede afirmar que la empresa reflejó tener una mayor madurez de capacidades tecnológicas que el centro de investigación. Lo anterior se muestra de forma gráfica en la Figura 5. 


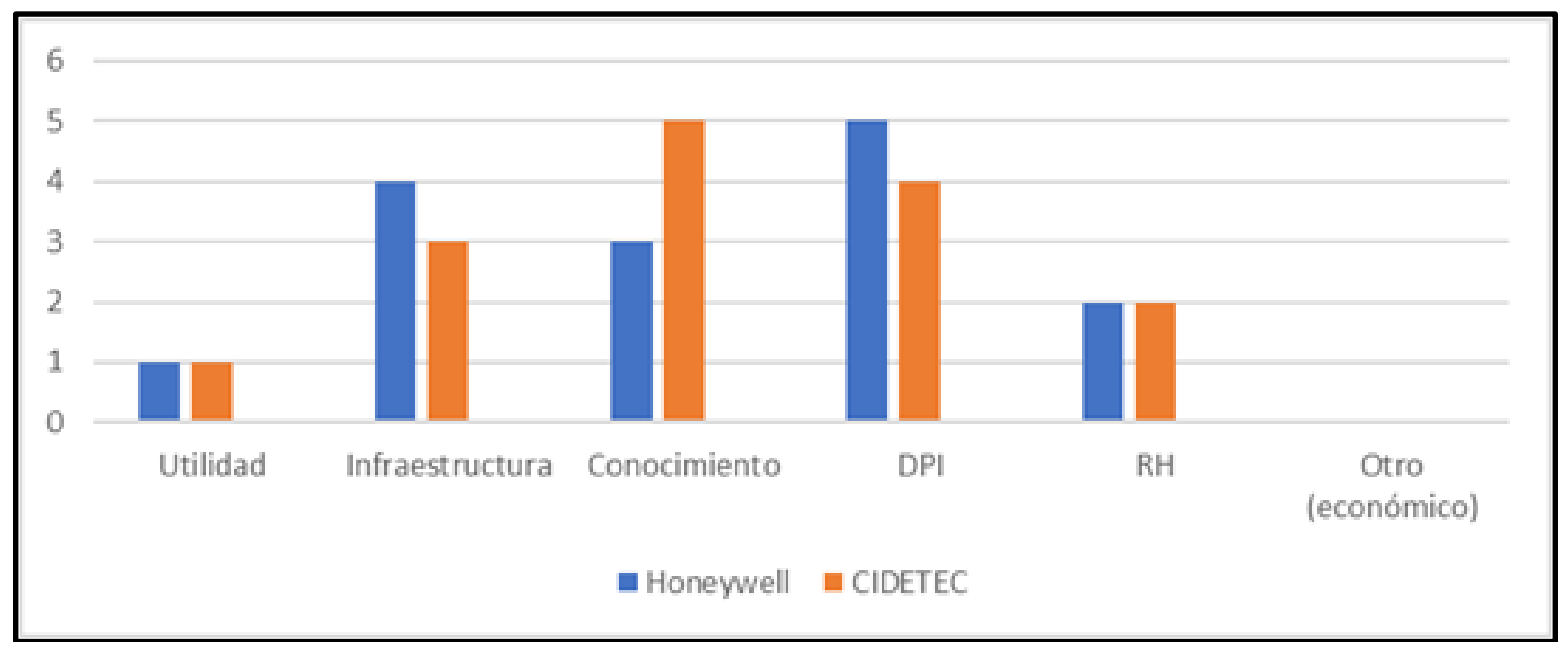

Figura 5. Factores que maximizan la vinculación

Figure 5. Factors that increase the likelihood of linkages Fuente: elaboración propia.

Nota: la escala es Likert, donde 5 el más importante y 1 el menos importante.

Por último, se indagaron las capacidades organizacionales (dimensiones) que fueron relevantes para establecer vinculaciones. Los resultados indican que la dimensión científica y tecnológica fue la más sobresaliente para ambas organizaciones. Después, la empresa consideró la dimensión administrativa como el segundo factor más importante, mientras que la dimensión económica fue el segundo en interés para Cidetec. Estos resultados sugieren que las distorsiones se pueden entender si se considera la necesidad de mayores recursos de la entidad pública (Cidetec) para consolidar capacidades que generen mayor conocimiento de valor económico, siendo éste un tema resuelto en organizaciones consolidadas e independientes económicamente como Honeywell.

Es importante resaltar el contraste entre ambas instituciones con respecto a la percepción del elemento organizacional, porque Honeywell lo consideró un factor crucial mientras que Cidetec lo valoró como de poco interés. Esto lleva a plantear la existencia de una contradicción de metas en el centro de investigación, especialmente si se considera que los recursos y capacidades (relativamente limitados) que se poseían al momento de la investigación no les generaban problemas para la vinculación mutua a los líderes de ambas organizaciones. Esta situación crea zonas de interpretación de empirismos aplicativos en las decisiones para aliarse. La Figura 6 reporta las valoraciones de la importancia de las capacidades organizacionales para cada una de las organizaciones analizadas desde la perspectiva de los lideres entrevistados. 


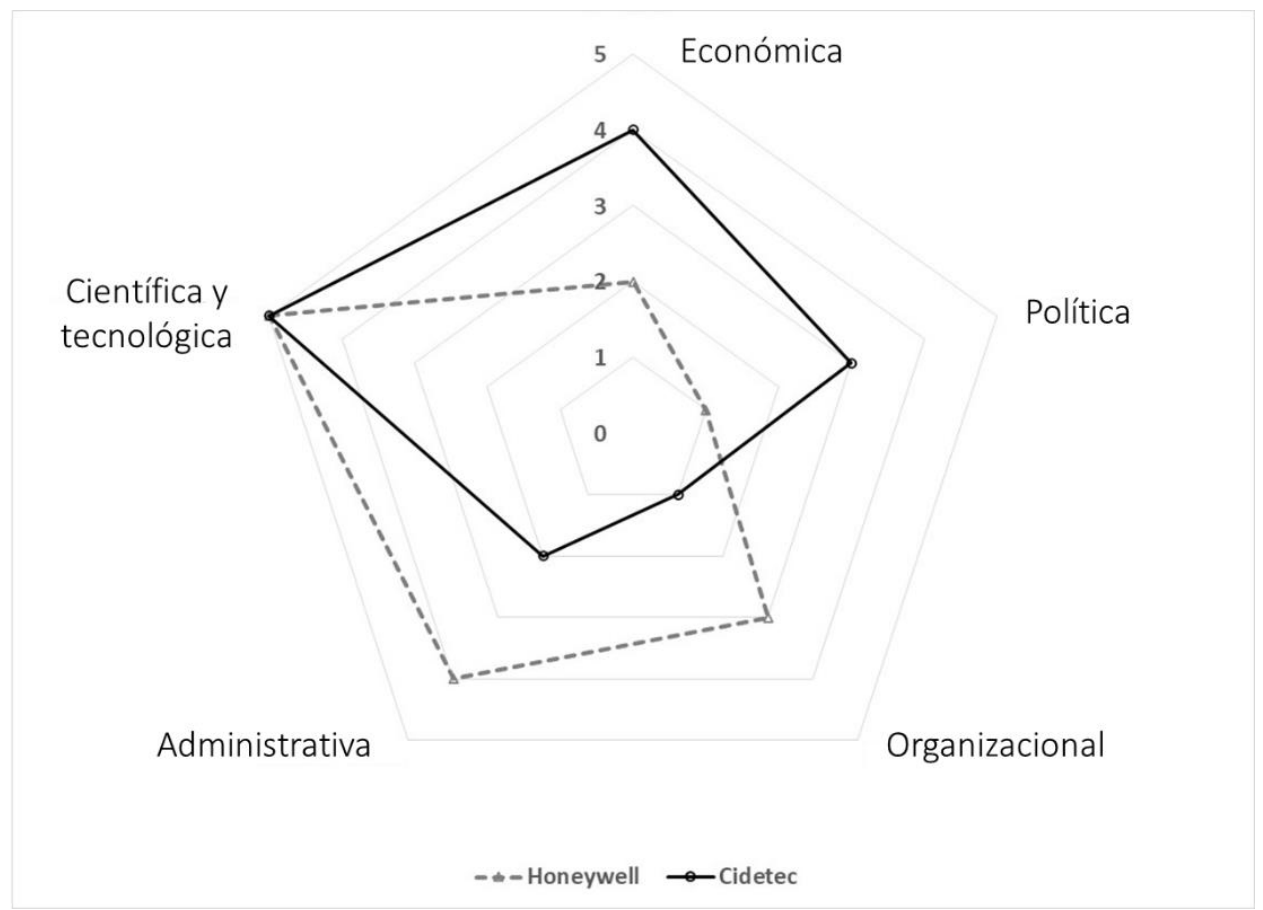

Figura 6. Importancia de las capacidades internas para la vinculación

Figure 6. Radar chart of the importance of internal capabilities for linkages

Fuente: elaboración propia.

Nota: la escala es Likert, donde 5 el más importante y 1 el menos importante.

\section{DISCUSIÓN}

Se analizarán ahora las coincidencias y divergencias del modelo de referencia propuesto para las variables de la realidad estudiadas (líderes y organizaciones), contrastándolas con los hallazgos documentales y de campo con la finalidad de identificar los efectos de las categorías de análisis, es decir, distorsiones, discrepancias y empirismos, usando las respuestas de los entrevistados.

Para empezar, la evidencia derivada de este estudio indica, siguiendo a Kogut (2008), que la organización empresarial buscó poseer capacidades más sólidas, mostrando interés en establecer alianzas para acrecentar su conocimiento científico y tecnológico, así como para explotar los derechos de propiedad intelectual desarrollados con antelación. Por otra parte, el centro de investigación buscó establecer alianzas para obtener recursos económicos al tener una actitud más endeble hacia el mercado, pero teniendo como activo un capital humano especializado. Esto refuerza la noción de que la interacción y dependencia de los recursos y capacidades organizacionales coincide con la propuesta teórica de Levinthal y Myatt (1994), puesto que las organizaciones buscan desarrollar elementos como la discreción gerencial, el aprendizaje organizacional y las actividades de retroalimentación con el mercado para mejorar sus capacidades de innovación. Dado lo anterior, es pertinente cuestionarse si las visiones de los líderes sufrieron de distorsiones, y de la información disponible se deduce que probablemente tendieron a favorecer la consolidación de algunas capacidades y la inhibición de otras; situación que también coincide con el modelo ya referido de coevolución de capacidades y de discreción gerencial de Levinthal y Myatt (1994). 
Con relación a los vacíos en la aplicación de teorías y conceptos (empirismos) por parte de estas organizaciones, a partir de los hallazgos se puede inferir que su existencia se debe a la probable ausencia de contactos con otras instituciones. Al parecer faltan vínculos con organizaciones intermedias, diseños y análisis organizacionales óptimos, implementación de sistemas de medición para la gestión de la tecnología y la innovación, mayor claridad en los procesos de generación de innovaciones, sensibilización de las racionalidades y de los efectos hacia los distintos niveles de una organización. La inexistencia de interacciones de vinculación con agentes intermedios por parte de las dos organizaciones analizadas es un hallazgo importante, pues, a pesar de que México cuenta con personas y entes que realizan las funciones de organizaciones intermedias, no se reportaron acciones formales de vinculación. No obstante, sí se observaron vínculos informales derivados de las relaciones de cercanía personal derivados del estilo de los liderazgos analizados (racionalidades). Otro vacío identificado fue con relación al diseño óptimo organizacional, lo que limita el desarrollo de nuevas capacidades para las dos instituciones bajo estudio.

Se observó también que Honeywell utiliza algunas herramientas del modelo teórico propuesto, reconociéndolas como necesarias. Esta organización también se destaca por la aplicación de técnicas más sofisticadas, entre las que sobresalen el método Kaizen, las evaluaciones periódicas y los estudios tecnológicos. Se concluye que los conceptos en los que se apoya una organización intensiva en conocimiento para establecer alianzas surgen de dimensiones internas e intermedias; lo que produce una colección de variables cualitativas para evaluar organizaciones deseosas de establecer alianzas para innovar. El primer factor (dimensión interna) está conformado por las variables de racionalidades, recursos y capacidades, análisis de los sistemas de acción concreta y el análisis organizacional. Estas son las causas más importantes de las distorsiones, discrepancias y empirismos que enfrentan las alianzas en el marco teórico referencial de este trabajo. Estos hallazgos respaldan la propuesta de Yasuda e lijima (2005) acerca de que la operatividad de las alianzas responde a elementos de tipo idiosincrático, donde la gestión incluye el efecto del aprendizaje, siendo crucial el papel que puedan jugar los líderes para incorporarlo. El segundo factor (dimensión intermedia), lo conforman las variables de alianzas, innovación tecnológica y organizaciones intermedias. En esta investigación se hallaron evidencias de distorsiones en las dos primeras variables, mientras que en la variable de organizaciones intermedias se encontraron marcados empirismos. Esto puede ser en parte explicado por los frágiles ecosistemas de innovación donde se mueven las organizaciones mexicanas, confirmando nuevamente la validez de los constructos de Yasuda e lijima (2005), ya que el dinamismo de la vinculación se refleja en la calidad del nivel de transferencia de conocimiento, los procedimientos de aprendizaje y los mecanismos para la protección intelectual emanados de la alianza.

Finalmente, el análisis efectuado se puede reforzar con herramientas de gestión de la tecnología y la innovación (Morcillo Ortega, 1997) y estudios prospectivos (Miklos \& Tello, 2008). En particular, Morcillo Ortega (1997) sugiere estudios tipo benchmarking, además de la evaluación de riesgos tecno-económicos para mejorar la cultura organizacional. Si bien las dos organizaciones hacen uso de algunas de ellas, el centro de investigación se mostró más alejado, mientras que la empresa tuvo mayor implementación. 


\section{CONCLUSIONES}

Las alianzas interorganizacionales son un recurso fundamental para el desempeño exitoso de muchas empresas y sectores industriales; no obstante, un número elevado de asociaciones no llega a satisfacer las expectativas de los participantes. Aunque el origen y causas de estos fracasos se deben a razones que van más allá de los motivos estrictamente organizacionales, un elemento común que subyace a estas fallas es la aplicación de estrategias deficientes surgidas de modelos teóricos inviables. Si bien el naufragio ocasional de alianzas interorganizacionales puede obedecer a una selección inapropiada de los socios a partir de una identificación deficiente de metas comunes, las fallas resultantes se podrían evitar mediante la participación informada de los líderes organizacionales, condición que debiera ejercerse en anticipación a la formalización de las posibles vinculaciones. Es de ahí que el análisis de los mecanismos que determinan las decisiones de las organizaciones para establecer alianzas estratégicas cobra una importancia crucial.

Por otra parte, en el caso de las alianzas entre organizaciones intensivas en conocimiento, la discusión sobre los factores que explican el fracaso de éstas también merece una revisión detallada puesto que presentan particularidades adicionales, como la influencia del nivel acumulado de conocimiento organizacional. Así, una alianza estratégica exitosa estará fuertemente determinada por el manejo eficiente de los llamados activos intangibles, donde el papel de los líderes es igualmente crucial.

Este trabajo analizó las alianzas entre organizaciones intensiva en conocimiento. Para tal fin, se acotó el alcance a la validación de opiniones vertidas por líderes de dos organizaciones radicadas en México (Honeywell \& Cidetec), pertenecientes a la rama electrónica. El análisis se apoyó en la evaluación del entorno organizacional donde el objeto de estudio fueron los líderes de estas organizaciones y su percepción de la realidad cotidiana, estimándose estas variables como causas del problema bajo investigación: la discrepancia observada entre las intenciones y las acciones ejecutadas por estos líderes en ambas organizaciones. En segundo lugar, se establecieron las variables del problema, establecidas como el efecto del fenómeno estudiado, estas fueron definidas como distorsiones, discrepancias y empirismos aplicativos. En tercer lugar, se trabajó en la construcción de un modelo teórico que permitiera abordar las variables de la realidad y relacionarlas con las variables del problema bajo dos dimensiones (o factores). El primero, denominado factor interno, con variables de racionalidad de liderazgos y los recursos y capacidades de las organizaciones; el segundo, ubicado como el factor intermedio, contemplando las variables que explican la realización de alianzas para la innovación. Finalmente se usaron tres elementos para medir el grado de construcción de las alianzas: 1) la gestión de la tecnología e innovación, 2) la dirección estratégica de la tecnología y 3) la innovación y prospectiva tecnológica. Estos tres elementos se consideraron estratégicos para la visualización del alcance y la construcción de las alianzas.

Los resultados arrojan interesantes datos empíricos para entender el rol del conocimiento especializado en la generación de productos tecnológicos, específicamente de componentes electrónicos. Del análisis documental y de las entrevistas se desprende que la principal motivación para establecer alianzas fue la construcción de sinergias para minimizar costos, así como la reducción de los tiempos de aprendizaje y producción. En particular, se encontró que el centro público de investigación (Cidetec) buscó aprovechar su dotación de recursos humanos calificados para obtener mayores recursos económicos, al tiempo que consolida el conocimiento acumulado. Por su parte, la empresa (Honeywell) buscó maximizar su portafolio de propiedad intelectual. Sin embargo, la 
conformación de alianzas adolece de serias deficiencias estructurales que surgen de factores como la falta de control para la implementación de sistemas de medición sobre manejo de las herramientas de gestión de la tecnología y la innovación, la falta de claridad en los objetivos de las innovaciones intentadas y la subutilización de la infraestructura disponible. Estas fallas obedecen a las distorsiones en las visiones y estrategias aplicadas por parte de los líderes en ambas organizaciones, lo que inhibe las capacidades para generar innovaciones tecnológicas. Asimismo, al tener orígenes y misiones distintos, se producen notorias discrepancias en los mecanismos de implementación de alianzas en las dos instituciones debido a una aplicación imprecisa de los conceptos y teorías para gestionar la complementariedad de recursos y capacidades.

Si bien se encontró evidencia de que los líderes tendieron a generar empirismos aplicativos, estos se podrían subsanar mediante un proceso de fertilización cruzada, en donde cada organización fuera capaz de ceder conocimientos y capacidades para compartir experiencias y procesos más efectivos en aras del aprendizaje mutuo. Empero, este paso es complejo pues los líderes tendieron a defender sus procesos de creación de capacidades para la innovación, negando muchas de las ventajas que la literatura ha documentado como derivadas de las alianzas entre organizaciones intensivas en conocimiento y tecnología, por lo que las estructuras y procesos actuales en estas dos organizaciones tienden a ralentizar sus procesos de vinculación para incentivar innovaciones.

Por último, proponemos que, para futuros estudios, se construyan indicadores que permitan hacer un análisis mixto de las alianzas entre organizaciones intensivas en conocimiento. Estos indicadores además servirán para identificar una colección de factores y variables explicativas más amplio y especializado por nicho tecnológico, lo que facilitaría la caracterización del tipo de organización de acuerdo con el caso de estudio que se pretenda aplicar. Debemos subrayar, sin embargo, que la literatura en estudios organizacionales aún carece de una propuesta teórica robusta para analizar el papel que juegan los líderes en la creación y sostenimiento de alianzas estratégicas, y por lo tanto aún no se cuenta con modelos que faciliten la replicabilidad entre casos de estudios.

\section{AGRADECIMIENTOS}

Los autores agradecen el apoyo del Programa de Doctorado en Gestión y Políticas de Innovación del Instituto Politécnico Nacional (IPN) de México para la realización de este trabajo, el cual también contó con recursos del proyecto de investigación SIP-20200079 del IPN.

\section{CONFLICTOS DE INTERÉS}

Los autores declaran que no presentan conflictos de interés financiero, profesional o personal que pueda influir de forma inapropiada en los resultados obtenidos o las interpretaciones propuestas.

\section{CONTRIBUCIÓN DE AUTORES}

Para el desarrollo de este proyecto todos los autores han realizado una contribución significativa especificada a continuación: 
Juan Carlos Becerril-Elías: preparación del esquema de investigación, elaboración del marco teórico y recolección de la evidencia empírica. Preparación de tablas, figuras y gráficas. Redacción general del manuscrito y conclusiones.

Humberto Merritt: revisión de la literatura, incluyendo citas y referencias bibliográficas. Análisis de los datos. Contribución a la redacción general del manuscrito a partir de la revisión final de la introducción, discusión y conclusiones.

\section{REFERENCIAS}

Albers, S.; Wohlgezogen, F.; Zajac, E. J. (2013). Strategic Alliance Structures: An Organization Design Perspective. Journal of Management, v. 42, n. 3, 582-614. https://doi.org/10.1177/0149206313488209

Álvarez, H. f. (2015). La convergencia de racionalidades en la dirección de organizaciones. Gestión Joven, n. 13, 125-147. URL

Barney, J. (1991). Firm resources and sustained competitive advantage. Journal of Management, v. 17, n. 1, 99-120. https://doi.org/10.1177/014920639101700108

Belso-Martínez, J. A.; Díez-Vial, I. (2018). Firm's Strategic Choices and Network Knowledge Dynamics: How Do They Affect Innovation? Journal of Knowledge Management, v. 22, n. 1, 1-20. https://doi.org/10.1108/JKM-12-2016-0524

Bouncken, R. B.; Plüschke, B. D.; Pesch, R.; Kraus, S. (2016). Entrepreneurial orientation in vertical alliances: joint product innovation and learning from allies. Review of Managerial Science, v. 10, n. 2, 381-409. https://doi.org/10.1007/s11846-014-0150-8

Bund, E.; Gerhard, U.; Hoelscher, M.; Mildenberger, G. (2015). A Methodological Framework for Measuring Social Innovation. Historische Sozialforschung, v. 40, n. 3, 48-78. https://doi.org/10.12759/hsr.40.2015.3.48-78

Caballero Romero, A. (2014). Metodología Integral Innovadora para Planes y Tesis. La metodología de cómo formularlos. Cengage Learning.

Centro de Innovación y Desarrollo Tecnológico en Cómputo [CIDETEC]. (2021). URL

Coletti, M.; Landoni, P. (2018). Collaborations for Innovation: A Meta-Study of Relevant Typologies, Governance and Policies. Economics of Innovation and New Technology, v. 27, n. 5-6, 493-509. https://doi.org/10.1080/10438599.2017.1376166

Collis, D. J. (1994). How Valuable Are Organizational Capabilities? Strategic Management Journal, v. 15, 143-152. URL 
Contractor, F. J.; Reuer, J. J. (2019). Frontiers of Strategic Alliance Research: Negotiating, Structuring and Governing Partnerships. Cambridge University Press.

Creswell, J. W.; Plano Clark, V. L. (2017). Designing and Conducting Mixed Methods Research. Sage.

Crozier, M.; Friedberg E. (1990). El actor y el sistema: las restricciones de la acción colectiva. Alianza Editorial.

De Man, A. P. (2014). Alliances: An Executive Guide to Designing Successful Strategic Partnerships. John Wiley \& Sons.

DePamphilis, D. M. (2018). Mergers, Acquisitions, and Other Restructuring Activities: An Integrated Approach to Process, Tools, Cases, and Solutions. Academic Press.

García-Canal, E.; Valdés-Llaneza, A.; Sánchez-Lorda, P. (2013). Ámbito de la Cooperación y Forma de Gobierno de las Alianzas Tecnológicas. Cuadernos de Economía y Dirección de la Empresa, v. 16, n. 2, 71-82. https://doi.org/10.1016/i.cede.2012.06.001

Gillespie, J.; Gulati, R. (2001). Alliances and Joint Ventures en Smelser, N. J. \& Baltes, P. B. (Eds.), International Encyclopedia of the Social and Behavioral Sciences (pp. 392-397). Pergamon.

Gulati, R. (2007). Managing Network Resources: Alliances, Affiliations, and Other Relational Assets. Oxford University Press.

Gulati, R.; Lavie, D.; Madhavan, R. (2011). How do networks matter? The performance effects of interorganizational networks. Research in Organizational Behavior, v. 31, 207-224. https://doi.org/10.1016/j.riob.2011.09.005

Haeussler, C.; Patzelt, H.; Zahra, S. A. (2012). Strategic Alliances and Product Development in High Technology New Firms: The Moderating Effect of Technological Capabilities. Journal of Business Venturing, v. 27, n. 2, 217-233. https://doi.org/10.1016/j.jbusvent.2010.10.002

Hagedoorn, J. (1993). Understanding the Rationale of Strategic Technology Partnering: Interorganizational Modes of Cooperation and Sectoral Differences. Strategic Management Journal, v. 14, n. 5, 371-385. https://doi.org/10.1002/smj.4250140505

Holmberg, S. R.; Cummings, J. L. (2009). Building Successful Strategic Alliances: Strategic Process and Analytical Tool for Selecting Partner Industries and Firms. Long Range Planning, v. 42, n. 2, 164193. https://doi.org/10.1016/i.Irp.2009.01.004.

Honeywell. (2021). Honeywell. URL

Kehrel, U.; Klischan, K.; Sick, N. (2016). Why Research Partnerships Fail in the Biotechnology SectorAn Empirical Analysis of Strategic Partnerships. International Journal of Innovation and Technology Management, v. 13, n. 1, 1650003. https://doi.org/10.1142/S0219877016500036 
Kogut, B. (2008). Knowledge, Options, and Institutions. Oxford University Press.

Levinthal, D. A.; Myatt, J. (1994). Co-Evolution of Capabilities and Industry: The Evolution of Mutual Fund Processing. Strategic Management Journal, v. 15, n. S1, 45-62. https://doi.org/10.1002/smj.4250150905

Marra, A.; Carlei, V.; Baldassari, C. (2020). Exploring networks of proximity for partner selection, firms' collaboration and knowledge exchange. The case of clean-tech industry. Business Strategy and the Environment, v. 29, n. 3, 1034-1044. https://doi.org/10.1002/bse.2415

Miklos, T.; Tello, M. E. (2008). Planeación prospectiva: una estrategia para el diseño del futuro. Limusa.

Morcillo Ortega, P. (1997). Dirección estratégica de la tecnología e innovación, un enfoque de competencias. Editorial Civitas.

Muwardi, D.; Saide, S.; Eko Indrajit, R.; Iqbal, M., Siti Astuti, E.; Herzavina, H. (2020). Intangible resources and institution performance: The concern of intellectual capital, employee performance, job satisfaction, and its impact on organization performance. International Journal of Innovation Management, v. 24, n. 5, 2150009. https://doi.org/10.1142/S1363919621500092

Nooteboom, B. (2010). A Cognitive Theory of the Firm: Learning, Governance and Dynamic Capabilities. Edward Elgar.

Pavitt, K. (2005). Innovation Processes, en Fagerberg, J., Mowery, D. C. y Nelson, R. R. (Eds.) The Oxford Handbook of Innovation (pp. 86-114). Oxford University Press.

Porter, M. E. (1990). The Competitive Advantage of Nations. The Free Press.

Prahalad, C. K.; Hamel, G. (1990). The Core Competence of the Corporation. Harvard Business Review, v. 68, n. $3,79-91$ URL

Teece, D. J.; Pisano, G. P.; Shuen, A. (1997). Dynamic Capabilities and Strategic Management. Strategic Management Journal, v. 18, n. 7, 509-533. URL

Tjemkes, B.; Vos, P.; Burgers, K. (2017). Strategic Alliance Management. Routledge.

Weng, C. S. (2017). Innovation Intermediaries in Technological Alliances. International Journal of Innovation and Technology Management, v. 14, n. 2, 1740013. https://doi.org/10.1142/S0219877017400132

Yang, W.; Meyer, K. E. (2019). Alliance Proactiveness and Firm Performance in an Emerging Economy. Industrial Marketing Management, v. 82, 226-237. https://doi.org/10.1016/j.indmarman.2019.01.010 
Yasuda, H.; lijima, J. (2005). Linkage Between Strategic Alliances and Firm's Business Strategy: The Case of Semiconductor Industry. Technovation, v. 25, n. 5, 513-521.

https://doi.org/10.1016/j.technovation.2003.09.005

Yoshino, M. Y.; Rangan, U. S. (1995). Strategic Alliances: An Entrepreneurial Approach to Globalization. Harvard Business Review Press.

Zhao, J.; Wu, G.; Xi, X.; Na, Q.; Liu, W. (2018). How Collaborative Innovation System in A KnowledgeIntensive Competitive Alliance Evolves: An Empirical Study on China, Korea and Germany. Technological Forecasting and Social Change, v. 137, 128-146.

https://doi.org/10.1016/j.techfore.2018.07.001 